\title{
Comprehensive and Equitable Care for Vulnerable Veterans With Integrated Palliative, Psychology, and Oncology Care
}

Joanna L Martin, MD; Desiree R. Azizoddin, PsyD; Lauren Z Rynar, PhD; Jane Weber, MSN, RN, FNP-BC; Tyra Oliver, LCSW, BCD; Christine B. Weldon, MBA; and Joshua M. Hauser, MD

Objective: Veterans who live with cancer need comprehensive care. The National Comprehensive Cancer Network and the American College of Surgeons Commission on Cancer guidelines recommend evaluating distress and providing appropriate follow-up to all patients with cancer.

Methods: We created patient-centered, collaborative clinics to screen for and address cancer-related distress. Medical oncologists received education about available supportive services and instructions on how to make referrals. Participants completed the Coleman Supportive Oncology Collaborative screening questions.

Results: Patients in this outpatient US Department of Veterans Affairs medical oncology clinic were primarily older, Af- rican American men. Most veterans screened positive for $\geq 1$ type of cancer-related distress. Patients screened for high levels of distress received in-person clinical follow-up for further evaluation and to make immediate referrals to supportive care services.

Conclusions: We evaluated patients' needs, made referrals as needed, and helped bring care directly into the oncology clinic. Using a screening tool for cancer-related distress and managing distress with integrated psychosocial providers could improve care coordination and enhance patient-centered supportive oncology care, especially for high-risk patients. A full-time social worker was integrated into the medical oncology clinics based on our program's success.
Author affiliations can be found at the end of the article. Correspondence: Joanna Martin (joanna.martin3@va.gov)

Fed Pract. 2021;38(suppl 3) Published online August 12 doi: $10.12788 / \mathrm{fp} .0158$
V eterans living with cancer need comprehensive assessment that includes supportive psychosocial care. The National Comprehensive Cancer Network (NCCN) and American College of Surgeons Commission on Cancer require accredited cancer centers to evaluate psychosocial distress and provide appropriate triage and treatment for all patients..$^{1-3}$ Implementing psychosocial distress screening can be difficult because of procedural barriers and time constraints, clinic and supportive care resources, and lack of knowledge about how to access supportive services.

Distress screening protocols must be designed to address the specific needs of each population. To improve screening for cancer-related distress, deliver effective supportive services, and gain agreement on distress screening standards of care, the Coleman Foundation supported development of the Coleman Supportive Oncology Collaborative (CSOC), a project of 135 interdisciplinary health care professionals from 25 Chicago-area cancer care institutions. ${ }^{4}$

The Jesse Brown US Department of Veterans Affairs (VA) Medical Center (JBVAMC) was chosen to assess cancer-related concerns among veterans using the $\mathrm{CSOC}$ screening tool and to improve access to supportive oncology. JBVAMC provides care to approximately 49,000 veterans in Chicago, Illinois, and northwestern Indiana. The JBVAMC patient popula- tion includes a large number of veterans with dual diagnoses (co-occurring substance use and mental health disorders) and veterans experiencing homelessness.

Delivering integrated screening and oncologic care that is culture and age appropriate is particularly important for veterans given their unique risk factors. The veteran population is considered vulnerable in terms of health status, psychological functioning, and social context. Veterans who use the VA health system as a principal source of care have poorer health, greater comorbid medical conditions, and an increased risk of mortality and suicide compared with the general population. ${ }^{5,6}$ Poorer health status in veterans also may relate to old age, low income, poor education, psychological health, and minority race. ${ }^{7-9}$

Past studies point to unique risk factors for cancer and poor cancer adjustment among veterans, which may complicate cancer treatment and end-of-life/survivorship care. Veteran-specific risk factors include militaryrelated exposures, particularly Agent Orange and morbidity/mortality secondary to comorbid medical and psychiatric conditions (eg, chronic obstructive pulmonary disease, diabetes mellitus, and posttraumatic stress disorder [PTSD])..$^{10-12}$ Moreover, the geriatric veteran population continues to grow, with increasing rates of cancer that require unique considerations for effective cancer care. ${ }^{13,14}$ Despite this, there are minimal data to inform best 
practices and supportive care approaches for veterans with cancer. Lack of guidelines specific to veterans and other populations with increased psychosocial challenges may impede successful cancer care, making distress screening procedures particularly important. This is especially the case for the JBVAMC, which serves primarily African American urbandwelling veterans who experience high rates of cancer disparities, including increased rates of mortality and increased levels of psychosocial distress. ${ }^{15,16}$

The goals of this program were to (1) examine levels of psychological, physical, financial, and treatment-related distress in a large sample of urban-dwelling veterans; (2) create a streamlined, sustainable process to screen a large number of veterans receiving cancer care in the outpatient setting and connect them with available supportive services; and (3) educate oncology physicians, nurses, and other staff about cancerrelated distress and concerns using in-service trainings and interpersonal interactions to improve patient care. Our program was based on a Primary Care Mental Health Integration (PCMHI) model that embeds health psychologists in general medical clinics to better reach veterans dealing with mental health issues. We tailored for palliative care involvement.

Studies of this model have shown that mental health integration improves access to mental health services and mental health treatment outcomes and has higher patient and provider satisfaction. ${ }^{17}$ We were also influenced by the construct of the patient aligned care team (PACT) social worker who, in this veterancentered approach, often functions as a care coordinator. Social work responsibilities include assessment of patients' stressors including adjusting to the medical conditions, identifying untreated or undertreated mental health or substance abuse issues, economic instability, legal problems, and inadequate housing and transportation, which can often be exacerbated during cancer treatment. ${ }^{18}$

We screened for distress-related needs that included mental health concerns, physical needs including uncontrolled symptoms or adverse effects of cancer treatment, physical function complaints (eg, pain and fatigue), nutrition concerns, treatment or care related concerns, family and caregiver needs, along with financial challenges (housing and food) and insurance-related support. The goal of this article is to describe the de-
TABLE 1 Patient Demographics

\begin{tabular}{|c|c|}
\hline Characteristics & Results \\
\hline Age, mean [range] (SD), y & 71.8 [36-99] (9.5) \\
\hline Sex, male, No. (\%) & $556(96.3)$ \\
\hline \multicolumn{2}{|l|}{ Cancer diagnosis, No. $(\%)^{a}$} \\
\hline Lung & 125 (21.3) \\
\hline Prostate & $97(16.6)$ \\
\hline Breast & $56(9.6)$ \\
\hline Multiple myeloma & $47(8.0)$ \\
\hline Ear, nose, and throat & $33(5.6)$ \\
\hline Lymphoma & $33(5.6)$ \\
\hline Leukemia & $29(4.9)$ \\
\hline Colorectal & $27(4.6)$ \\
\hline Gastric & $24(4.1)$ \\
\hline Pancreatic & $17(2.9)$ \\
\hline \multicolumn{2}{|l|}{ Cancer stage, No. (\%) } \\
\hline । & $54(9.2)$ \\
\hline II & $45(7.7)$ \\
\hline III & $74(12.6)$ \\
\hline IV & $197(33.6)$ \\
\hline \multicolumn{2}{|l|}{ Race, No. (\%) } \\
\hline African American & $412(70.3)$ \\
\hline White & $137(23.4)$ \\
\hline Other & $9(1.5)$ \\
\hline
\end{tabular}

aOther cancer diagnoses included, No. (\%): urinary 12(2.0); hepatocellular 11 (1.9); melanoma 4 (0.7); renal 19 (3.2); other 43 (7.3); and thyroid $1(0.2)$.

velopment and implementation of this VA-specific distress screening program and reflect on the lessons learned for the application of streamlined distress screening and triage in similar settings throughout the VA health system and other similar settings.

\section{METHODS}

This institutional review board at JBVAMC reviewed and exempted this quality improvement program using the SQUIRE framework..$^{19}$ It was led by a group of palliative care clinicians, psychologists, and administrators who have worked with the oncology service for many years, primarily in the care of hospitalized patients. Common palliative care services include providing care for patients with serious illness diagnosis through the illness trajectory.

\section{Setting}

At the start of this program, we assessed the current clinic workflow to determine how to best screen and assist veterans experiencing distress. We met with team members individually to identify the best method of clinic integration, including attending medical oncologists, medical oncology fellows, psychology interns, oncology nursing staff, the oncology nurse coordinator, and clinic clerks. 
The JBVAMC provides cancer care through 4 half-day medical hematology-oncology clinics that serve about 50 patients per half-day clinic. The clinics are staffed by hematologyoncology fellows supervised by hematologyoncology attending physicians, who are affiliated with 2 academic medical centers. These clinics are staffed by 3 registered nurses (RNs) and a licensed practical nurse (LPN) and are adjacent to a chemotherapy infusion clinic with unique nursing staff. The JBVAMC also provides a variety of supportive care services, including extensive mental health and substance use treatment, physical and occupational therapy, acupuncture, nutrition, social work, and housing services. Following our assessment, it was evident that there were a low number of referrals from oncology clinics to supportive care services, mostly due to lack of knowledge of resources and unclear referral procedures.

Based on clinical volume, we determined that our screening program could best be implemented through a stepped approach beginning in one clinic and expanding thereafter. We began by having a palliative care physician and health psychology intern embedded in 1 weekly halfday clinic and a health psychology intern embedded in a second weekly half-day clinic. Our program included 2 health psychology interns (for each academic year of the program) who were supervised by a JBVA health psychologist.

About 15 months after successful integration within the first 2 half-day clinics, we expanded the screening program to staff an additional half-day medical oncology clinic with a palliative care APRN. This allowed us to expand the screening tool distribution and collection to 3 of 4 of the weekly half-day oncology clinics as well as to meet individually with veterans experiencing high levels of distress. Veterans were flagged as having high distress levels by either the results of their completed screening tool or by referral from a medical oncology physician. We initially established screening in clinics that were sufficiently staffed to ensure that screens were appropriately distributed and reviewed. Patients seen in nonparticipating clinics were referred to outpatient social work, mental health and/or outpatient palliative care according to oncology fellows' clinical assessments of the patient. All oncology fellows received education about distress screening and methods for referring to supportive care. Our clinic screening pro- gram extended from February 2017 through January 2020.

\section{Screening}

Program staff screened patients with new cancer diagnoses, then identified patients for followup screens. This tracking allowed staff to identify patients with oncology appointments that day and cross-reference patients needing a followup screen.

Following feedback from the clinic nurses, we determined that nurses would provide the distress tool to patients in paper form after they completed their assessment of vitals and waited to be seen by their medical oncologist. The patient would then deliver their completed form to the nurse who would combine it with the patient's clinic notes for the oncologist to review. Veterans who reported elevated 4-question Patient Health Questionnaire (PHQ4) scores $\geq 6$ were seen immediately by program staff. Veterans were referred to social work or psychiatry services for a same day visit if they endorsed a high level of psychological distress during clinical examination. They were referred for other supportive care services if they were determined to have practical, family, or nutrition unmet distress needs by either the program staff or oncology fellows. Program staff provided guidance to medical oncology fellows for needed referrals including social work, mental health, and palliative care follow ups (eAppendix A available at doi:10.12788 /fp.0158).

Veterans referred for supportive care services were contacted by the relevant clinical administrator by phone to schedule an intake; for social work referrals, patients were either seen in a walk-in office located in a colocated building or contacted by a social worker by phone.

Our screening tool was the Coleman Foundation Supportive Oncology Collaborative Screening Tool, compiled from validated instruments. Patients completed this screening tool, which includes the PHQ-4, NCCN problem list concerns, adapted Mini Nutrition Assessment and PROMIS Pain and Fatigue measure (eAppendix B available at doi:10.12788/fp.0158). ${ }^{20-22}$

We also worked with the VA Computerized Patient Record System (CPRS) to create an electronic template for the screening tool. Completed screening tools were manually entered by the physician, psychologists, or APRN into the CPRS chart. 
We analyzed the different supportive care services available at the JBVAMC and noticed that many supportive services were available, yet these services were often separated. Therefore, we created a consult flowsheet to assist oncologists in placing referrals. These supportive care services include mental health services, a cancer support group, home health care, social services, nutrition, physical medicine and rehabilitation, and other specialty services.

\section{Patient Education}

The psychology and nursing staff created a patient information bulletin board where patients could access information about supportive services available at JBVAMC. This board required frequent replenishment of handouts because patients consulted the board regularly. Handouts and folders about common clinical issues also were placed in the clinic treatment rooms. We partnered with 2 local cancer support centers, Gilda's Club and the Cancer Support Center, to make referrals for family members and/ or caregivers who would benefit from additional support.

We provided in-service trainings for oncology fellows, including trainings on PTSD and substance abuse and their relationship to cancer care at the VA. These topics were chosen based on the feedback program staff received about perceived knowledge gaps from the oncology fellows. This program allowed for multiple informal conversations between that program staff and oncology fellows about overall patient care. We held trainings with the cancer coordinator and clinical nursing staff on strategies to identify and follow-up on cancer-related distress, and with oncology fellows to review the importance of distress screening and to instruct fellows on instructions for the consult flowsheet.

\section{Funding}

This program was funded by the Chicagobased Coleman Foundation as part of the CSOC. Funding was used to support a portion of time for administrative and clinical work of program staff, as well as data collection and analysis.

\section{RESULTS}

We established 3 half-day integrated clinics where patients were screened and referred for services based on supportive oncology needs. In addition to our primary activities to screen
TABLE 2 Prevalence of Supportive Care Needs

\begin{tabular}{|c|c|}
\hline Types of Supportive Care & Results \\
\hline \multicolumn{2}{|l|}{ Practical concerns, No. $(\%)^{\mathrm{a}, 1}$} \\
\hline Child care & $11(1.9)$ \\
\hline Paying for food & $57(9.7)$ \\
\hline Paying for housing & $61(10.4)$ \\
\hline Transportation & $109(18.6)$ \\
\hline Work/school & 27 (4.6) \\
\hline Insurance coverage & 70 (11.9) \\
\hline Paying for medical care & $53(9.0)$ \\
\hline Living alone & $164(28.0)$ \\
\hline \multicolumn{2}{|l|}{ Self-care, No. $(\%)^{a}$} \\
\hline Confined to bed & $97(16.6)$ \\
\hline Help with chores & $119(20.3)$ \\
\hline Help with errands & $126(21.5)$ \\
\hline Family concerns, No. (\%) ${ }^{\mathrm{a}}$ & $120(20.5)$ \\
\hline Concerns about children & $65(11.1)$ \\
\hline Concerns about partner & $85(14.5)$ \\
\hline Concern about caregiver & $61(10.4)$ \\
\hline Ability to have children & $20(3.4)$ \\
\hline Concerns about other family issues & $120(20.5)$ \\
\hline \multicolumn{2}{|l|}{ Physical concerns, No. (\%) ${ }^{a}$} \\
\hline Breathing & $160(27.3)$ \\
\hline Constipation & $143(24.4)$ \\
\hline Diarrhea & $88(15.0)$ \\
\hline Fever & $21(3.6)$ \\
\hline Nausea and vomiting & $68(11.6)$ \\
\hline Sleep & $239(40.8)$ \\
\hline Changes in urination & 114 (19.5) \\
\hline Difficulty chewing & $77(13.1)$ \\
\hline Mouth sore & $28(4.8)$ \\
\hline Dry mouth & $179(30.5)$ \\
\hline Dental/teeth & $151(25.8)$ \\
\hline Cough & 101 (17.2) \\
\hline Swollen arm or leg & $140(23.9)$ \\
\hline Feeling full & $92(15.7)$ \\
\hline Sex intimacy & $161(27.5)$ \\
\hline Dry skin & $162(27.6)$ \\
\hline Tingling in hands and feet & $207(35.3)$ \\
\hline Appearance & 66 (11.3) \\
\hline Tobacco/cigarettes/vaping & 64 (10.9) \\
\hline Alcohol/drugs & $35(6.0)$ \\
\hline Difficulty concentrating & $108(18.4)$ \\
\hline Difficulty remembering & $178(30.4)$ \\
\hline Difficulty finding words & $86(14.7)$ \\
\hline Other & $23(3.9)$ \\
\hline
\end{tabular}

\begin{tabular}{ll}
\hline${\text { Nutrition, No. }(\%)^{\mathrm{b}}}$ & \\
Weight loss/lack of appetite $^{\text {Weight gain }}$ & $180(30.7)$ \\
Issues with taste & $118(20.1)$ \\
Concern about nutritious food & $112(19.1)$ \\
\hline Treatment or care concerns, No. $(\%)^{\mathrm{b}}$ & $131(22.4)$ \\
Diagnosis/stage & \\
Prognosis & $395(67.4)$ \\
Treatment options & $418(71.3)$ \\
Discussion other (combine option and medical care 2 variables) & $323(55.1)$ \\
\hline Pain score, mean (SD) & $189(32.3)$ \\
\hline Fatigue score, mean (SD) & \\
\hline
\end{tabular}

aBased on Patient Screening Questions for Supportive Care and include the National Comprehensive Cancer Network Guidelines for Distress Management Needs Assessment Problem list; scored with binary responses $(0$ or 1$)$ clarifying presence of symptoms. bMini Nutrition Assessment short-form and Treatment or Care Concerns scale binary response options (0 or 1 ).

cPROMIS National Institute of Health Pain and Fatigue 4-item measures (0-5 scale). 
TABLE 3 Successes and Barriers in a Veteran-Specific Distress Screening Implementation Program
Successes
- Established integrated care clinic with oncology, palliative care, psychology, and social work
- Created educational materials for patients in clinic lobby and rooms and infusion suites
- Created flow chart of available clinical services with instructions on referrals
- Increased patient referrals to supportive services with high rates of referrals to social work for financial/housing support, hospice, home health, and mental health care
- Partnered with private groups that provided art therapy and other supportive services, which was available to family and caregivers

\section{Barriers}
- Medical oncology fellows had primary training off-site, had limited experience with the US Department of Veterans Affairs, and were unfamiliar with referring to supportive services
- Lack of education of available supportive services that were available separately
- Electronic health record system limited ability to track patients referred to supportive services

the CSOC for depressed mood, pain, housing, transportation, and physical, nutrition, and treatment concerns. ${ }^{23}$ Elevated presence of needs was especially prominent for food, housing and insurance/medical needs; physical concerns; nutrition, and treatment- or care-related concerns. Veterans in this cohort reported extensive financial and housing concerns: $10.4 \%$ reported food and housing concerns, $18.6 \%$ reported transportation concerns, and $9.0 \%$ reported issues paying for medical care or medications (Table 2). ${ }^{20}$ Anecdotally, many experienced job loss or strain with their cancer diagnosis or were living at the poverty level before their diagnosis.

Social work referrals were often triggered due to transportation barriers to appointments/medication access, and food and/or housing insecurity. Social workers assisted with referrals for housing, transportation, financial reimbursement, on-site or community-based food banks, home health support, familial support, and hospice services. Social work consults increased $166 \%$ from 2016 (the year before the program start date) to the end of 2019.

Based on this increased volume of referrals for social work in our oncology clinics, an oncologyspecific social worker was hired at the completion of our program to be based in all 4 half-day oncology clinics in response to results of our quality improvement intervention. The social worker currently sees all patients with a new cancer diagnosis and supports oncology fellows to identify veterans needing a palliative care referral or referrals to other supportive services.

Throughout program implementation, traditional areas of palliative care focus were particularly important as veterans reported significant concerns with understanding their illness $(67.4 \%)$, wanting to understand their prognosis (71.3\%), and having questions about their treatment options $(55.1 \%) .^{20}$ The palliative care providers spent time educating patients about their disease, coordinating goals of care conversations, promoting patients' engagement in decision making, and making a large number of referrals to hospice and home health to support veterans at home. with mental health and social work services have been reported elsewhere. ${ }^{23}$

The mean (SD) age for veterans in this cohort was 72 (9.5) years. Participants were primarily African American veterans ( $70 \%)$, with mostly advanced disease (Table 1). Participants endorsed elevated distress needs compared with other patient populations screened in Chicago through

\section{DISCUSSION}

This project created a successful program to screen veterans for psychosocial distress and triage them to appropriate services. During the project, patients in VA-outpatient oncology clinics reported significant cancer-related distress 
due to baseline psychosocial needs, changes in emotional and physical functioning, logistical and financial challenges of receiving cancer care, and lack of instrumental support. ${ }^{23}$

Staff education supported successful buy-in, development and implementation of supportive oncology programs. We used a combination of in-service trainings, online trainings, and handouts to provide evidence for distress screening. ${ }^{24}$ Highlighting the evidence-base that demonstrates how cancer-related distress screening improves cancer and quality of life outcomes helped to address physician reluctance to accept the additional requirements needed to address veterans' psychosocial needs and care concerns. To increase buy-in and collaboration among team members and foster heightened understanding between providers and patients, we recommend creating accessible education for all staff levels.

One specific area of education we focused on was primary palliative care, which includes the core competencies of communication and symptom management recommended for generalists and specialists of all disciplines. ${ }^{25}$ Program staff supported oncology fellows in developing their primary palliative care skills by being available to discuss basic symptom management and communication issues. VA cancer care programs could benefit from ongoing palliative care education of oncology staff to facilitate primary palliative care as well as earlier integration of secondary palliative care when needed. ${ }^{26} \mathrm{Sec}-$ ondary palliative care or care provided directly by the palliative care team assists with complex symptom management or communication issues. For these needs, oncology fellows were encouraged to refer to either the palliative care staff available in one of the half-day clinics or to the outpatient palliative care clinic. As a unique strength, the VA allows veterans to receive concurrent cancer-directed therapy and hospice care, which enables earlier referrals to hospice care and higher quality end-of-life care and emphasizes the need for primary palliative care in oncology. ${ }^{27,28}$

Integrating supportive oncology team members, such as licensed clinical social worker and psychology interns, was successful. This was modeled on the VA PACT, which focuses on prevention, health promotion, coordination and chronic disease management. ${ }^{29}$ Social determinants of health have a major impact on health outcomes especially in veteran-specific and Afri- can American populations, making screening for distress critical. ${ }^{30-32}$ The VA Office of Health Equity actively addresses health inequities by supporting initiation of screening programs for social determinants of health, including education, employment, exposure to abuse and violence, food insecurity, housing instability, legal needs, social isolation, transportation needs, and utility needs. This is especially needed for African-American individuals who are not only more likely to experience cancer, but also more likely to be negatively impacted by the consequences of cancer diagnosis/treatment, such as complications related to one's job security, access to care, adverse effects, and other highly distressing needs. . $^{33,34}$

Our program found that veterans with cancer often had concerns associated with food and housing insecurity, transportation and paying for medication or medical care, and screening allowed health care providers to detect and address these social determinants of health through referrals to VA and community-specific programs. Social workers integrated into VA cancer clinics are uniquely equipped to coordinate distress screening and support continuity of care by virtue of their training, connections to preexisting VA supportive services, and knowledge of community resources. This model could be used in other VA specialty clinics serving veterans with chronic illness and those with high levels of physical frailty. ${ }^{35}$

Our ability to roll out distress screening was scaffolded by technological integration into existing VA systems (eg, screening results in CPRS and electronic referrals). Screening procedures could have been even more efficient with improved technology (Table 3). For example, technological limitations made it challenging to easily identify patients due for screening, requiring a cumbersome process of tracking, collecting and entering patients' paper forms. Health care providers seeking to develop a distress screening program should consider investing in technology that allows for identification of patients requiring screening at a predetermined interval, completion of screening via tablet or personal device, integration of screening responses into the electronic health record, and automatic generation of notifications to the treating physician and appropriate support services.

We also established partnerships with community cancer support groups to offer both referral pathways and in-house programming. Veterans' cancer care programs could benefit 
from identifying and securing community partnerships to capitalize on readily available lowcost or no-cost options for supportive oncology in the community. Further, as was the case in our program, cancer support centers may be willing to collaborate with VA hospitals to provide services on site (eg, support groups, art therapy). This would extend the reach of these supportive services while allowing VA employees to address the extensive psychosocial needs of individual veterans.

\section{CONCLUSIONS}

Veterans with cancer benefited from enhanced screening and psychosocial service availability, similar to a PCMHI model. Robust screening programs helped advocate for veterans dealing with the effects of poverty through identification of need and referral to existing VA programs and services quickly and efficiently. Providing comprehensive care within ambulatory cancer clinics can address cancer-related distress and any potential barriers to care in real time. VA hospitals typically offer an array of supportive services to address veterans' psychosocial needs, yet these services tend to be siloed. Integrated referrals can help to resolve such access barriers. Since many veterans with burdensome cancers are not able to see their VA primary care physician regularly, offering comprehensive care within medical oncology ensures complete and integrated care that includes psychosocial screening.

We believe that this program is an example of a mechanism for oncologists and palliative care clinicians to integrate their care in a way that identifies needs and triages services for vulnerable veterans. As colleagues have written, "it is fundamental to our commitment to veterans that we ensure comparable, high quality care regardless of a veteran's gender, race, or where they live." ${ }^{34}$ Health care providers may underestimate the extensive change a cancer diagnosis can have on a patient's quality of life. Cancer diagnosis and treatment have a large impact on all individuals, but this impact may be greater for individuals in poverty due to inability to work from home, inflexible work hours, and limited support structures. By creating screening programs with psychosocial integration in oncology clinics such as we have described, we hope to improve access to more equitable care for vulnerable veterans.
Author affiliations

Joanna Martin and Joshua Hauser are Palliative Care Physicians; Jane Weber is a Palliative Care Nurse Practitioner; and Tyra Oliver is a Palliative Care and Hematology Oncology Clinical Social Worker; all at Jesse Brown VA Medical Center in Chicago, Illinois. Christine Weldon is Adjunct Faculty in Hematology and Oncology; Joanna Martin is a Health System Clinician; and Joshua Hauser is a Palliative Care Physician; all at Northwestern Feinberg School of Medicine in Illinois. Christine Weldon is Director at the Center for Business Models in Healthcare in Illinois. Desiree Azizoddin is a Research Scientist at Brigham and Women's Hospital and Affiliate Research Faculty, Department of Psychosocial Oncology and Palliative Care, Dana Farber Cancer Institute, and Harvard Medical School, in Massachusetts. Lauren Rynar is an Assistant Professor, Supportive Oncology at Rush University Medical Center in Chicago.

\section{Author disclosures}

The authors report no actual or potential conflicts of interest with regard to this article.

\section{Disclaimer}

The opinions expressed herein are those of the authors and do not necessarily reflect those of Federal Practitioner, Frontline Medical Communications Inc., the US Government, or any of its agencies.

\section{References}

1. National Comprehensive Cancer Network. NCCN guidelines distress management. Version 2.2021. Updated January 5, 2021. Accessed July 8, 2021. http://www.nccn.org /professionals/physician_gls/pdf/distress.pdf

2. American College of Surgeons, Commission on Cancer. Cancer program standards 2012: ensuring patient-centered care. Version 1.2.1. Published 2021. Accessed July 8, 2021. https://www.facs.org/ /media/files/quality\%20 programs/cancer/coc/programstandards2012.ashx

3. Jacobsen PB, Ransom S. Implementation of NCCN distress management guidelines by member institutions. J Natl Compr Canc Netw. 2007;5(1):99-103. doi:10.6004/jnccn.2007.0010

4. The Coleman Supportive Oncology Collaborative. Training tools. Accessed July 14, 2021. https://www .supportiveoncologycollaborative.org/training-tools

5. Agha Z, Lofgren RP, VanRuiswyk JV, Layde PM. Are patients at Veterans Affairs medical centers sicker? A comparative analysis of health status and medical resource use. Arch Intern Med. 2000;160(21):3252-3257. doi:10.1001/archinte.160.21.3252

6. Bullman T, Schneiderman A, Gradus JL. Relative importance of posttraumatic stress disorder and depression in predicting risk of suicide among a cohort of Vietnam veterans. Suicide Life Threat Behav. 2019;49(3):838-845. doi:10.1111/sltb.12482

7. Kazis LE, Miller DR, Clark J, et al. Health-related quality of life in patients served by the Department of Veterans Affairs: results from the Veterans Health Study. Arch Intern Med. 1998;158(6):626-632. doi:10.1001/archinte.158.6.626

8. O'Toole BI, Marshall RP, Grayson DA, et al. The Australian Vietnam Veterans Health Study: III. Psychological health of Australian Vietnam veterans and its relationship to combat. Int J Epidemiol. 1996;25(2):331-340. doi:10.1093/ije/25.2.331

9. Vincent C, Chamberlain K, Long N. Mental and physical health status in a community sample of New Zealand Vietnam War veterans. Aust J Public Health. 1994;18(1):58-62. doi:10.1111/j.1753-6405.1994.tb00196.x

10. US Department of Veterans Affairs. Veterans' diseases associated with Agent Orange. Updated June 16, 2021. Accessed July 8, 2021. http://www.publichealth.va.gov /exposures/agentorange/diseases.asp\#veterans

11. Hwa KJ, Dua MM, Wren SM, Visser BC. Missing the obvi- 
ous: psychosocial obstacles in Veterans with hepatocellular carcinoma. HBP (Oxford). 2015;17(12):1124-1129. doi:10.1111/hpb.12508

12. Saha S, Freeman M, Toure J, Tippens KM, Weeks C, Ibrahim S. Racial and ethnic disparities in the VA health care system: a systematic review. J Gen Intern Med. 2008;23(5):654-671. doi:10.1007/s11606-008-0521-4

13. Amaral EFL, Pollard MS, Mendelsohn J, Cefalu M. Current and future demographics of the veteran population, 2014-2024. Popul Rev. 2018;57(1):28-60. doi:10.1353/prv.2018.0002

14. Mohile SG, Dale W, Somerfield MR, et al. Practical assessment and management of vulnerabilities in older patients receiving chemotherapy: ASCO guideline for geriatric oncology. J Clin Oncol. 2018;36(22):2326-2347. doi:10.1200/JCO.2018.78.8687

15. Siegel R, Ward E, Brawley O, Jemal A. Cancer statistics, 2011: the impact of eliminating socioeconomic and racial disparities on premature cancer deaths. CA Cancer J Clin. 2011;61(4):212-236. doi:10.3322/caac.20121

16. Cimino T, Said K, Safier L, Harris H, Kinderman A. Psychosocial distress among oncology patients in the safety net. Psychooncology. 2020;29(11):1927-1935. doi:10.1002/pon.5525

17. Molander R, Hodgkins K, Johnson C, White A, Frazier E, Krahn D. Interprofessional education in patient aligned care team primary care-mental health integration. Fed Pract. 2017;34(6):40-48.

18. Parikh DA, Ragavan $M$, Dutta $R$, et al. Financial toxicity of cancer care: an analysis of financial burden in three distinct health care systems [published online ahead of print, 2021 Apr 7]. JCO Oncol Pract. 2021;OP2000890. doi:10.1200/OP.20.00890

19. Ogrinc G, Davies L, Goodman D, Batalden P, Davidoff F, Stevens D. SQUIRE 2.0 (Standards for QUality Improvement Reporting Excellence): revised publication guidelines from a detailed consensus process. BMJ Qual Saf. 2016;25(12):986-992. doi:10.1136/bmjqs-2015-004411

20. Weldon CB, Gerhart JI, Penedo FJ, et al. Correlates of distress for cancer patients: results from multiinstitution use of holistic patient-reported screening tool. J Clin Oncol. 2019;37(15)(suppl):11587-11587. doi:10.1200/JCO.2019.37.15_suppl.11587

21. Kroenke K, Spitzer RL, Williams JB, Löwe B. The Patient Health Questionnaire Somatic, Anxiety, and Depressive Symptom Scales: a systematic review. Gen Hosp Psychiatry. 2010;32(4):345-359. doi:10.1016/j.genhosppsych.2010.03.006

22. Kaiser MJ, Bauer JM, Ramsch $C$, et al. Validation of the Mini Nutritional Assessment short-form (MNA-SF): a practical tool for identification of nutritional status. J Nutr Health Aging. 2009;13(9):782-788. doi:10.1007/s12603-009-0214-7

23. Azizoddin DR, Lakin JR, Hauser J, et al. Meeting the guidelines: implementing a distress screening intervention for veterans with cancer. Psychooncology. 2020;29(12):2067-2074. doi:10.1002/pon.5565

24. Carlson LE, Waller A, Mitchell AJ. Screening for distress and unmet needs in patients with cancer: review and recommendations. J Clin Oncol. 2012;30(11):1160-1177. doi:10.1200/JCO.2011.39.5509

25. Quill TE, Abernethy AP. Generalist plus specialist palliative care-creating a more sustainable model. N Engl J Med. 2013;368(13):1173-1175. doi:10.1056/NEJMp1215620

26. Weissman DE, Meier DE. Identifying patients in need of a palliative care assessment in the hospital setting: a consensus report from the Center to Advance Palliative Care. J Palliat Med. 2011;14(1):17-23. doi:10.1089/jpm.2010.0347

27. Kumar P, Wright AA, Hatfield LA, Temel JS, Keating NL. Family perspectives on hospice care experiences of patients with cancer. J Clin Oncol. 2017;35(4):432-439. doi:10.1200/JCO.2016.68.9257

28. Mor V, Joyce NR, Coté DL, et al. The rise of concurrent care for veterans with advanced cancer at the end of life. Cancer. 2016;122(5):782-790. doi:10.1002/cncr.29827

29. US Department of Veterans Affairs. Patient care services: Patient aligned care team (PACT). Updated November 5, 2020. Accessed July 8, 2021. https://www.patientcare .va.gov/primarycare/PACT.asp

30. US Department of Veterans Affairs, Veterans Health Administration. VHA health equity action plan. Published September 27, 2019. Accessed July 8, 2021. https://www .va.gov/HEALTHEQUITY/docs/Health_Equity_Action Plan_Final_022020.pdf

31. Alcaraz KI, Wiedt TL, Daniels EC, Yabroff KR, Guerra CE, Wender RC. Understanding and addressing social determinants to advance cancer health equity in the United States: a blueprint for practice, research, and policy. $C A$ Cancer J Clin. 2020;70(1):31-46. doi:10.3322/caac.21586

32. Atkins D, Kilbourne A, Lipson L. Health equity research in the Veterans Health Administration: we've come far but aren't there yet. Am J Public Health. 2014;104(suppl 4):S525-526. doi:10.2105/AJPH.2014.302216

33. American Cancer Society. Cancer Facts \& Figures for African Americans 2019-2021. Atlanta: American Cancer Society; 2019.

34. Hastert TA, Kirchhoff AC, Banegas MP, et al. Work changes and individual, cancer-related, and work-related predictors of decreased work participation among African American cancer survivors. Cancer Med. 2020;9(23):9168-9177. doi:10.1002/cam4.3512

35. Bekelman DB, Nowels CT, Allen LA, Shakar S, Kutner JS, Matlock DD. Outpatient palliative care for chronic heart failure: a case series. J Palliat Med. 2011;14(7):815-821. doi:10.1089/jpm.2010.050 